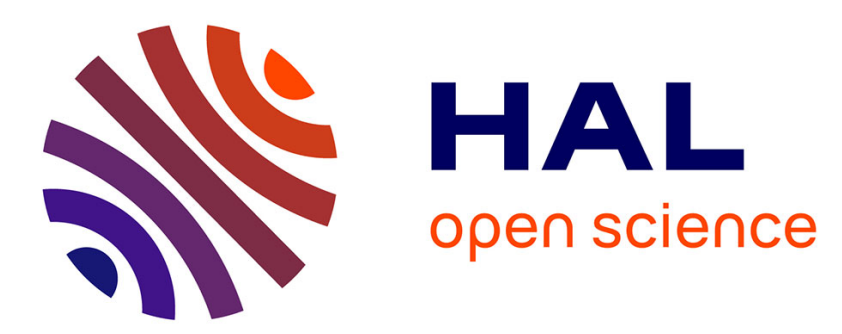

\title{
Canal des provisions bancaires et cyclicité du marché du crédit
}

\author{
Vincent Bouvatier, Laetitia Lepetit
}

\section{To cite this version:}

Vincent Bouvatier, Laetitia Lepetit. Canal des provisions bancaires et cyclicité du marché du crédit. Canadian Journal of Economics / Revue Canadienne d'Économique, 2011, 62 (1), pp.67. 10.3917/reco.621.0067 . hal-00785400

\section{HAL Id: hal-00785400 \\ https://hal-unilim.archives-ouvertes.fr/hal-00785400}

Submitted on 12 Nov 2014

HAL is a multi-disciplinary open access archive for the deposit and dissemination of scientific research documents, whether they are published or not. The documents may come from teaching and research institutions in France or abroad, or from public or private research centers.
L'archive ouverte pluridisciplinaire HAL, est destinée au dépôt et à la diffusion de documents scientifiques de niveau recherche, publiés ou non, émanant des établissements d'enseignement et de recherche français ou étrangers, des laboratoires publics ou privés. 


\title{
Canal des provisions bancaires et cyclicité
}

\author{
du marché du crédit*
}

\author{
Vincent Bouvatier ${ }^{\dagger} \quad$ Laetitia Lepetit ${ }^{\ddagger}$
}

Résumé : Des travaux empiriques portant sur les comportements de provisionnement des banques montrent que les provisions pour pertes évoluent de façon contracyclique. Ce fait stylisé est intégré dans un modèle théorique déterminant le comportement d'une banque représentative sur le marché du crédit. Le modèle et les simulations réalisées montrent que les modifications du coût de provisionnement supportées par la banque à travers un cycle économique amplifient les fluctuations sur le marché du crédit. Deux mécanismes sont identifiés pour éliminer cette effet d'amplification. D'un point de vue réglementaire, l'adoption d'un système de provisionnement dynamique représente une première solution. Au niveau de la banque, la constitution d'un "coussin de sécurité" en capital bancaire représente une seconde solution.

Abstract :The literature on provisioning bank behaviour shows that loan loss provisions are counter-cyclical. Based on this stylised fact, this paper develops a partial equilibrium model of a banking firm that analyzes how provisioning rules influence credit market fluctuations. The model and the simulations show that a backward-looking provisioning system amplifies the fluctuations in the credit market over an economic cycle. Two solutions are proposed to remove this bank provision channel. First, the regulatory authority can implement a forward-looking provisioning system. Second, if a backwardlooking provisioning system rules are implemented, banks can build a capital buffer to cover the expected losses which are not covered by loan loss reserves.

Classification JEL : G21

Mots clés : marché du crédit, provisions pour pertes

*Les auteurs souhaitent remercier Carlos Bautista, Christian Bordes, Thérèse Chevallier-Farat, Falko Fetch, Antoine Faure-Grimaud, Andy Mullineux, Alain Sauviat, Amine Tarazi et Clas Wihlborg pour leurs différentes remarques et suggestions. La responsabilité de toutes les erreurs demeure celle des auteurs.

†Université de Rennes 1, CREM - CNRS (UMR 6211), Faculté des Sciences Economiques, 7 place Hoche, 35065 Rennes Cedex, France. E-mail: vincent.bouvatier@univ-rennes1.fr

${ }^{\ddagger}$ Corresponding author: Université de Limoges, LAPE, 5 rue Félix Eboué, 87031 Limoges Cedex, France. Tel: +33-555-14-92-13, e-mail: laetitia.lepetit@unilim.fr 


\section{Introduction}

Nous avons pu constater au cours de ces vingts dernières années que les facteurs financiers, en particulier ceux associés au canal large du crédit, jouent un rôle croissant dans la propagation des cycles d'activités. L'identification des facteurs qui déterminent les fluctuations de court terme (ou cycliques) du marché du crédit revête ainsi toute son importance pour les banques centrales et les régulateurs bancaires dans la mesure où une excessive cyclicité sur le marché du crédit pourrait avoir des répercussionsnon seulement sur la stabilité macroéconomique mais aussi financière. Les fluctuations de court terme se produisant sur le marché du crédit ne peuvent pas être appréhendées intégralement par des modifications de la demande de crédits. Un certain nombre d'autres facteurs doivent également être considérés. Tout d'abord, des modifications du comportement des banques à travers le cycle économique, c'est-à-dire des facteurs d'offre, peuvent contribuer à expliquer ces fluctuations de court terme. Des théories sur le comportement des banques comme, par exemple, la myopie au désastre (Guttentag et Herring, 1986), le comportement moutonnier (Rajan, 1994) ou encore l'hypothèse de la mémoire institutionnelle (Berger et Udell, 2004) permettent de justifier des modifications du comportement des banques à travers le cycle économique. D'un point de vue empirique, certains travaux (Asea et Blomberg (1998) ou Lown et Morgan (2006) par exemple) montrent que, d'une part, les conditions auxquelles les banques accordent des crédits évoluent de façon cyclique et, d'autre part, que ces évolutions ont des répercussions sur l'ampleur à la fois du cycle du crédit et du cycle de l'activité économique. Ensuite, la réglementation bancaire peut représenter un autre facteur important dans la détermination des fluctuations de court terme se produisant sur le marché du crédit. Van den Heuvel (2002) définit, par exemple, un canal du capital bancaire dans lequel la contrainte réglementaire en fonds propres amplifie les 
effets des décisions de politique monétaire du fait d'imperfections sur le marché des capitaux propres bancaires. Par ailleurs, Zicchino (2006) montre que la réglementation sur les fonds propres ne conduit pas simplement à amplifier les chocs de politique monétaire mais plus généralement les chocs affectant les conditions macroéconomiques.

L'objectif de cet article est de montrer qu'un autre facteur d'ordre réglementaire peut contribuer à expliquer le phénomène d'amplification financière. Nous mettons en évidence, à l'aide d'un modèle théorique en équilibre partiel, que la réglementation sur le provisionnement pour pertes joue un rôle dans la détermination de l'ampleur des fluctuations cycliques se produisant sur le marché du crédit. A notre connaissance, aucun modèle ne permet de rendre compte de l'effet sur le marché du crédit de la réglementation sur les provisions pour pertes.

Le principal fait stylisé caractérisant les provisions pour pertes constituées par les banques est associé au caractère contracyclique fortement prononcé de leurs évolutions. Les banques sont en effet amenées à consistuer leurs provisions principalement suite à l'identification de prêts non performants, ces derniers se réalisant principalement pendant la phase basse du cycle de l'activité économique ${ }^{1}$. Laeven et Majnoni (2003) et Bikker et Metzemakers (2005) montrent, par exemple, que les provisions pour pertes constituées par les banques sont affectées négativement par le taux de croissance de la production. Borio et al. (2001), Clerc et al. (2001) et Fernandez de Lis et al. (2001) font apparaître également que le ratio entre les provisions pour pertes et le total des crédits évolue de façon fortement contracyclique dans de nombreux pays. Dans un tel contexte, les systèmes

\footnotetext{
${ }^{1}$ Dans la plupart des pays, les provisions pour pertes sont composées de provisions générales et de provisions spécifiques. Les provisions générales devraient couvrir les pertes attendues (ou pertes potentielles) mais aucune méthode précise ne définit la façon dont les banques doivent calculer leurs provisions générales. Les provisions spécifiques couvrent les prêts non performants identifiés par les banques, c'est à dire la partie avérée des pertes potentielles. Les évolutions contracycliques des provisions pour pertes observées renvoient donc principalement à des variations des provisions spécifiques qui dépendent ellesmêmes de l'identification des prêts non performants.
} 
de provisionnement sont qualifiés d'ex post. Nous interprétons cette contracyclicité des provisions pour pertes comme une imparfaite reconnaissance des pertes attendues (ou pertes potentielles) résultant de l'activité de crédit des banques.

Les pertes attendues sont définies par rapport à la moyenne de la distribution des pertes de crédit subies par une banque sur un cycle économique complet. Elles correspondent au taux de perte moyen pouvant être attendu. Elles existent donc à partir du moment où les prêts sont accordés. Par conséquent, une perte attendue peut être anticipée sur un prêt performant et devrait nécessiter la constitution d'une provision pour perte $^{2}$. Dans un système de provisionnement ex post, les pertes attendues sont couvertes principalement pendant la phase basse du cycle économique quand la majorité des prêts non performants est identifiée. Pendant la phase haute du cycle économique, nous pouvons ainsi définir un écart négatif entre les provisions pour pertes constituées dans un système de provisionnement ex post et les provisions pour pertes requises pour couvrir les pertes attendues. Inversement, cet écart devient positif pendant la phase de ralentissement économique quand les banques doivent constituer d'importantes provisions pour pertes pour faire face à l'augmentation des prêts non performants.

Les coûts résultant de l'activité de crédit et, par conséquent, les profits réalisés par les banques sont directement affectés par les évolutions contracycliques des provisions pour pertes. Un système de provisionnement ex post peut ainsi rendre le comportement d'offre de crédit de la banque plus sensible au cycle de l'activité économique. Plus précisément, nous définissons dans cet article un canal des provisions bancaires basé sur l'imparfaite reconnaissance des pertes attendues. Pendant la phase haute du cycle économique, les coûts liés à l'activité de crédit pourraient apparaître relativement faibles

\footnotetext{
${ }^{2}$ Plus précisément, les banques peuvent estimer leurs pertes attendues sur un cycle économique complet et en déduire une perte attendue moyenne par période qui requiert d'être provisionnée.
} 
du fait de l'amélioration de la qualité du portefeuille de crédit et donc de la flaiblesse des provisions pour pertes constituées par les banques. Les réactions des banques pourraient consister à relacher les conditions auxquelles elles accordent des crédits et à distribuer une grande partie de leurs profits sous forme de dividendes. Inversement, une détérioration de la conjoncture économique conduirait les banques à durcir les conditions auxquelles elles accordent des crédits.

Le canal des provisions bancaires est directement relié à l'effet cyclique des provisions pour pertes sur les coûts de l'activité de crédit. Par conséquent, toutes les mesures permettant de réduire l'écart entre les provisions pour pertes constituées dans un système de provisionnement ex post et les provisions pour pertes requises pour couvrir les pertes attendues permet d'atténuer l'ampleur du canal des provisions bancaires. Dans cet article, nous considérons deux possibilités pour atténuer l'ampleur de ce canal. Tout d'abord, d'un point de vue réglementaire, le régulateur bancaire pourrait modifier la réglementation sur les provisions pour pertes afin de mettre en oeuvre un système de provisionnement ex ante. Dans un tel système, les provisions pour pertes dépendent directement des pertes attendues et non plus des prêts non performants, conduisant à un lissage dans le temps des provisions pour pertes constituées par les banques ${ }^{3}$. Ensuite, au niveau de la banque, la constitution d'un "coussin de sécurité" en capital bancaire (capital buffer) pourrait permettre de prendre en compte la variabilité de la qualité du portefeuille de crédits à travers un cycle économique.

La suite est organisée de la façon suivante : la section 2 présente le modèle théorique, la section 3 démontre l'existence d'un canal des provisions bancaires et propose deux

\footnotetext{
${ }^{3}$ Voir Fernandez de Lis et al. (2001), Mann et Michael (2002) et Jiménez et Saurina (2006) pour plus de détails sur les systèmes de provisionnement ex ante. Un tel système a été mis en oeuvre en Espagne à partir de 2000 .
} 
alternatives pour atténuer ce canal, la section 4 présente le calibrage du modèle et les résultats des simulations et la section 5 conclut.

\section{Modélisation de la firme bancaire}

Nous développons un modèle en équilibre partiel caractérisant le comportement d'une firme bancaire ayant une activité traditionnelle d'intermédiation. Plus précisément, nous considérons une firme bancaire représentative de type $i(i=1, \ldots, N)$ évoluant sur un marché en concurrence monopolistique. La différenciation entre les banques porte sur les types de crédits qu'elles accordent. La banque se spécialise dans un certain type de prêts (pour les petites ou les grandes entreprises ou pour un secteur particulier) ou sur une certaine zone géographique (Carletti et al., 2007). Nous supposons également que la banque considère le cycle de l'activité économique comme exogène et que la réglementation en vigueur lui impose d'appliquer des règles de provisionnement ex post, c'est-à-dire que les provisions pour pertes dépendent essentiellement des prêts non performants.

\subsection{Origine et répartition du profit de la banque}

Les banques effectuent une activité d'intermédiation traditionnelle. L'actif de la banque $i$ est ainsi constitué d'un portefeuille de crédits $\left(L_{i, t}\right)$. Dans la mesure où le portefeuille de crédits contient des pertes attendues (ou pertes potentielles), les réserves pour pertes $\left(L L R_{i, t}\right)$, représentant le stock de provisions pour pertes accumulé par la banque, sont déduites de l'actif de la banque (Walter, 1991). L'actif enregistre ainsi la valeur nette du portefeuille de crédit de la banque. Par ailleurs, le passif de la banque est constitué de 
$\operatorname{dettes}^{4}\left(D_{i, t}\right)$ et de fonds propres $\left(K_{i, t}\right)$. Le bilan est donc défini par :

$$
L_{i, t}-L L R_{i, t}=D_{i, t}+K_{i, t}
$$

Le profit réalisé par la banque $i\left(\Pi_{i, t}\right)$ dépend de son activité d'intermédiation. Nous avons ainsi :

$$
\Pi_{i, t}=r_{i, t}^{L} L_{i, t}\left(1-\mathrm{J}\left(y_{t}\right)-\mathrm{G}\left(y_{t}\right)\right)-r_{t}^{M} D_{i, t}-L L P_{i, t}-\delta L_{i, t} \mathrm{G}\left(y_{t}\right),
$$

où $r_{i, t}^{L}$ est le taux d'intérêt sur les crédits, $r_{t}^{M}$ est le taux d'intérêt sur les dettes, $y_{t}$ est la production, les fonctions $\mathrm{J}\left(y_{t}\right)$ et $\mathrm{G}\left(y_{t}\right)$ représentent respectivement la proportion des prêts non performants et la proportion des prêts passés en charges ${ }^{5}, L L P_{i, t}$ représente les provisions pour pertes et $\delta_{t} L_{i, t} \mathrm{G}\left(y_{t}\right)$ représente la part non anticipée des passations en charges (i.e. les passations en charges pour lesquelles la banque n'a pas constitué de réserve pour pertes $)^{6}$. Les proportions des prêts non performants et des passations en charges sont définies par :

$$
\begin{aligned}
& \mathrm{J}\left(y_{t}\right)=j_{0}\left(y_{t} / y\right)^{-\omega}, \\
& \mathrm{G}\left(y_{t}\right)=g_{0}\left(y_{t} / y\right)^{-\theta},
\end{aligned}
$$

\footnotetext{
${ }^{4}$ Les dettes bancaires regroupent des dépôts ainsi que des financements de marché comme dans le modèle de Van den Heuvel (2002). De plus, nous supposons que toutes les dettes bancaires sont complètement assurées et que la prime d'assurance est égale à zéro. L'introduction explicite d'une prime d'assurance n'affecterait pas les résultats associés au comportement de provisionnement de la banque.

${ }^{5}$ Un prêt est passé en charges quand la banque reconnait qu'il ne sera par collecté. Il est ainsi retiré du bilan et correspond à une perte reconnue par la banque.

${ }^{6}$ Sur une période, le portefeuille de crédits de la banque contient trois catégories de prêts : $(i)$ les prêts performants, (ii) les prêts non performants et $(i i i)$ les prêts passés en charges. La majeure partie du portefeuille de crédits de la banque est constituée de prêts performants. Les prêts jugés susceptibles de ne pas être recouvrés sont classifiés comme prêts non performants. La passation en charges d'un prêt se produit lorsque la banque estime qu'un prêt est irrécouvrable en totalité ou en partie, ou qu'il n'existe aucune perspective sérieuse de recouvrement. La majeure partie des prêts passés en charges était classifiée comme prêts non performants sur la période précédente mais certains prêts peuvent directement passer de la catégorie $(i)$ à la catégorie $(i i i)$. Si la banque n'a pas accumulé de provisions pour ces prêts, ils correspondent à la part non anticipée des prêts passés en charges.
} 
où $j_{0}$ et $g_{0}$ sont respectivement les proportions à l'état stationnaire (ou les proportions moyennes sur un cycle économique complet) de prêts non performants et de passations en charges par période, $y_{t} / y$ représente l'output gap, $\theta$ et $\omega$ sont les élasticités par rapport à l'output gap $(\theta>0$ et $\omega>0)$. L'output gap affecte la situation financière des entreprises et des ménages et a donc un effet négatif sur les proportions des prêts non performants et des passations en charges.

L'équation (2) montre que les profits de la banque proviennent des intérêts reçus sur les crédits. Cependant, la banque ne reçoit des intérêts que sur une proportion (1 $\left.\mathrm{J}\left(y_{t}\right)-\mathrm{G}\left(y_{t}\right)\right)$ de son portefeuille de crédit, les prêts non performants et les passations en charges ne donnant pas lieu au versement d'intérêts. La proportion $(1-\delta)$ des passations en charges a été provisionnée durant les périodes précédentes et n'affecte donc pas le profit courant de la banque. Au contraire, la partie non anticipée des passations en charges $\left(\delta L_{i, t} \mathrm{G}\left(y_{t}\right)\right)$ représente une perte reconnue et non attendue par la banque et diminue donc son profit courant. La rémunération des dettes $\left(r_{t}^{M} D_{i, t}\right)$ et la constitution de provisions pour pertes $\left(L L P_{i, t}\right)$ viennent également diminuer les profits réalisés par la banque. La répartition du profit de la banque est donnée par :

$$
\Pi_{i, t}=K_{i, t+1}-K_{i, t}+\Delta_{i, t}
$$

où $\Delta_{i, t}$ représente les dividendes distribués aux actionnaires. La banque utilise donc une partie de son profit pour modifier ses fonds propres $\left(K_{i, t+1}-K_{i, t}\right)$ et l'autre partie est distribuée aux actionnaires. Des dividendes négatifs peuvent donc être interprétés comme une émission de fonds propres, impliquant que les dividendes peuvent être utilisés afin de 
respecter la contrainte réglementaire sur les fonds propres définie par :

$$
K_{i, t+1} \geqslant k_{0} L_{i, t+1}
$$

où $k_{0}$ représente le seuil de capital réglementaire (ce seuil étant fixé à $4 \%$ pour le TIER1 par les accords de Bâle I) et la pondération du risque sur les crédits est égale à 1 . Nous supposons que cette contrainte est toujours saturée, c'est-à-dire que les banques détiennent le minimum réglementaire de fonds propres ${ }^{7}$. Par ailleurs, la contrainte réglementaire de la banque est écrite pour la période $t+1$. La banque débute la période avec un montant donné de fonds propres $K_{i, t}$ et la part du profit qui n'est pas distribuée en dividendes permet de définir les fonds propres disponibles pour la période suivante.

\subsection{Réserves pour pertes et provisions pour pertes}

Les réserves pour pertes permettent d'ajuster la valeur du portefeuille de crédits en fonction des pertes potentielles. Sans cet ajustement dans l'actif de la banque, les responsables de la banque, les créditeurs de la banque, les régulateurs et les investisseurs auraient une vision tronquée de la rentabilité potentielle de la banque. La loi d'accumulation des réserves pour pertes est donnée par :

$$
L L R_{i, t+1}=L L R_{i, t}+L L P_{i, t}-L_{i, t} \mathrm{G}\left(y_{t}\right)+\delta L_{i, t} \mathrm{G}\left(y_{t}\right)
$$

\footnotetext{
${ }^{7}$ Cette hypothèse permet d'orienter notre analyse sur les effets des règles de provisionnement. Un prolongement de ce travail pourrait consister à prendre en compte également les effets du ratio de solvabilité sur le comportement de la banque. Il faudrait ainsi définir les coûts supportés par la banque lorsqu'elle se rapproche de la saturation de la contrainte sur les fonds propres et les coûts liés à l'émission de fonds propres comme par exemple dans le modèle de Furfine (2001).
} 
Les prêts passés en charges $\left(L_{i, t} \mathrm{G}\left(y_{t}\right)\right)$ sont soustraits des réserves pour pertes dans la mesure où ils représentent des pertes reconnues par la banque. Cependant, les prêts passés en charges n'ont pas été complètement provisionnés pendant les périodes précédentes. La partie non attendue des prêts passés en charges $\delta L_{i, t} \mathrm{G}\left(y_{t}\right)$ (prélevées sur les profits dans l'équation (2)) est donc également ajoutée au stock déjà existant de réserves pour pertes afin de maintenir les réserves pour pertes à un niveau suffisant pour absorber les pertes potentielles identifiées non encore reconnues (Walter, 1991). Les provisions pour pertes $L L P_{i, t}$ (prélevées sur les profits dans l'équation (2)) sont également ajoutées au stock déjà existant de réserves pour pertes.

Nous supposons que les règles de provisionnement que doivent appliquer les banques sont strictement ex post, c'est-à-dire que les provisions pour pertes se composent uniquement de provisions spécifiques $\left(\mathrm{H}^{\mathrm{S}}\left(L_{i, t}, y_{t}\right)\right)$. Ces dernières dépendent des prêts non performants, et elles couvrent donc une partie des prêts non performants identifiés plutôt que les pertes potentielles sur l'ensemble du portefeuille de crédits. Les provisions pour pertes sont ainsi définies par :

$$
L L P_{i, t}=\mathrm{H}^{\mathrm{S}}\left(L_{i, t}, y_{t}\right)=h_{0} L_{i, t} \mathrm{~J}\left(y_{t}\right)
$$

où $h_{0}$ représente la proportion à l'état stationnaire (ou la proportion moyenne calculée sur un cycle économique complet) du portefeuille de prêts non performants $\left(L_{i, t} \mathrm{~J}\left(y_{t}\right)\right)$ qui est provisionnée sur une période.

La spécification retenue assure une évolution contracyclique des provisions pour pertes. Cette caractéristique représente le principal fait stylisé spécifique à un système de provisionnement ex post (Clerc et al., 2001; Arpa et al., 2001; Fernandez de Lis et al., 
2001; Pain, 2003) $)^{8}$ Un provisionnement principalement basé sur l'identification des prêts non performants sous-estime les pertes potentielles pendant la phase haute du cycle économique et conduit les banques à devoir constituer d'importantes provisions pendant la phase basse du cycle. Ce système de provisionnement a donc un effet procyclique sur les profits des banques.

\subsection{Demande spécifique de crédit}

Nous supposons que les banques sont en concurrence monopolistique et qu'elles se différencient au niveau des types de crédits offerts. Chaque banque fait donc face à une demande de crédit spécifique. Nous supposons que cette demande spécifique est donnée par (Carletti et al., 2007 ; Henzel et al., 2007) :

$$
L_{i, t}=\left(\frac{r_{i, t}^{L}}{r_{t}^{L}}\right)^{-\zeta} \mu L_{t}
$$

où $L_{t}$ est le niveau agrégé des crédits, $r_{t}^{L}$ est le taux d'intérêt moyen sur les crédits, $\zeta>1$ est l'élasticité au taux d'intérêt de la demande de crédit s'adressant à la banque $i$ et $\mu$ est la part de marché de la banque (avec $\mu=1 / N)$. Le paramètre $\zeta$ représente donc une mesure inverse du pouvoir monopolistique de la banque.

\footnotetext{
${ }^{8}$ Nous ne considérons pas les provisions générales dans notre modèle dans la mesure où elles ne sont pas nécessaires pour reproduire une évolution contracyclique des provisions pour pertes.
} 


\subsection{Comportement de maximisation de la banque}

La banque maximise la somme actualisée de ses dividendes. Le problème de maximisation de la banque consiste à choisir son taux d'intérêt débiteur $\left(r_{i, t}^{L}\right)$ :

$$
\max _{r_{i, t}^{L}} \mathrm{E}_{t} \sum_{j=0}^{\infty} \beta^{t+j} \Delta_{t+j}
$$

où

$$
\Delta_{t}=r_{i, t}^{L} L_{i, t}\left(1-\mathrm{J}\left(y_{t}\right)-\mathrm{G}\left(y_{t}\right)\right)-r_{t}^{M} D_{i, t}-L L P_{i, t}-\delta L_{i, t} \mathrm{G}\left(y_{t}\right)+K_{i, t}-K_{i, t+1}
$$

sous la contrainte des équations (1), (3), (4), (6), (7), (8) et (9), et où $\beta$ est le paramètre d'actualisation standard.

La condition du premier ordre montre comment la banque fixe le taux d'intérêt sur les crédits ${ }^{9}$ :

$$
\begin{aligned}
r_{i, t}^{L}\left(1-\mathrm{J}\left(y_{t}\right)-\mathrm{G}\left(y_{t}\right)\right)= & \frac{\zeta}{\zeta-1}\left\{r_{t}^{M}\left(1-k_{0}\right)+\mathrm{E}_{t} \sum_{n=0}^{+\infty} \beta^{n+1} r_{t+1+n}^{M}\left[(1-\delta) \mathrm{G}\left(y_{t+n}\right)-h_{0} \mathrm{~J}\left(y_{t+n}\right)\right]\right. \\
& \left.+h_{0} \mathrm{~J}\left(y_{t}\right)+\delta \mathrm{G}\left(y_{t}\right)+\left(k_{0} / \beta-k_{0}\right)\right\} .
\end{aligned}
$$

Le membre de gauche de l'équation (12) représente le rendement marginal de l'activité de crédit étant donné que seule une proportion $\left(1-\mathrm{J}\left(y_{t}\right)-\mathrm{G}\left(y_{t}\right)\right)$ du portefeuille de crédits donne lieu à un versement d'intérêt. Le membre de droite de l'équation (12) montre que la banque fixe son taux d'intérêt en fonction de son pouvoir de marché et des différents coûts supportés. Plus précisément, compte tenu de sa position en concurrence monopolistique, la banque applique une marge (mark-up) de $\zeta /(\zeta-1)$ au coût marginal

\footnotetext{
${ }^{9}$ Les détails de l'ensemble des calculs sont disponibles auprès des auteurs.
} 
supporté.

\section{Canal des provisions bancaires}

Le modèle théorique développé précédemment est utilisé pour étudier l'impact des règles de provisionnement pour pertes sur le comportement des banques sur le marché du crédit. Nous montrons qu'un système de provisionnement ex post contribue à amplifier les fluctuations de court terme sur le marché du crédit. Ce canal des provisions bancaires peut être atténué par la mise en place d'un système de provisionnement dynamique (ou ex ante) ou la constitution d'un capital buffer par les banques.

\subsection{Amplifications des fluctuations de court terme sur le marché}

\section{du crédit dans un système de provisionnement ex-post}

Nous procédons à la log-linéarisation autour de son état stationnaire de la CPO associée au choix du taux d'intérêt sur les crédits (12) afin de déterminer l'effet du cycle économique sur le comportement de la banque ${ }^{10}$ :

$$
\varphi_{0} \hat{r}_{t}^{L}=\varphi_{0} \beta \mathrm{E}_{t}\left\{\hat{r}_{t+1}^{L}\right\}+\varphi_{1}\left(\hat{r}_{t}^{M}-\beta \mathrm{E}_{t}\left\{\hat{r}_{t+1}^{M}\right\}\right)-\varphi_{2}\left(\hat{y}_{t}-\beta \mathrm{E}_{t}\left\{\hat{y}_{t+1}\right\}\right)-L \hat{L} R_{t},
$$

où $\varphi_{0} \equiv r^{L}\left(1-j_{0}-g_{0}\right), \varphi_{1} \equiv Z r^{M}\left(1-k_{0}\right), \varphi_{2} \equiv \varphi_{2}^{A}+\varphi_{2}^{B}$ avec $\varphi_{2}^{A} \equiv \omega\left(r^{L} j_{0}+Z h_{0} j_{0}\right)$, $\varphi_{2}^{B} \equiv \theta\left(r^{L} g_{0}+Z \delta g_{0}\right)$ et $Z \equiv \zeta /(\zeta-1)$. De plus $L \hat{L} R_{t} \equiv \varphi_{3} \hat{y}_{t}$ où $\varphi_{3} \equiv \varphi_{3}^{A}+\varphi_{3}^{B}$ avec $\varphi_{3}^{A} \equiv-\omega\left(Z \beta r^{M} h_{0} j_{0}\right)$ et $\varphi_{3}^{B} \equiv \theta\left(Z \beta r^{M}(1-\delta) g_{0}\right)$.

L'équation (13) montre que les déviations du taux d'intérêt sur les crédits $\left(\hat{r}_{t}^{L}\right)$ dépen-

\footnotetext{
${ }^{10} \mathrm{La} \log$-linéarisation est effectuée à l'équilibre symétrique $\left(r_{i, t}^{L}=r_{t}^{L}\right)$. La variable $\hat{X}_{t}=\log \left(X_{t} / X\right)$ représente la déviation logarithmique de la variable $X_{t}$ par rapport à son état stationnaire $X$.
} 
dent d'une part des déviations du taux d'intérêt sur les dettes $\left(\hat{r}_{t}^{M}\right)$. Les banques reportent les modifications des coûts de financement qu'elles subissent sur le taux d'intérêt qu'elles pratiquent. D'autre part, le cycle économique $\left(\hat{y}_{t}\right)$ a un effet négatif sur les déviations du taux des crédits mesuré par le coefficient $\varphi_{2}$. Les différents paramètres qui composent ce coefficient renvoient à l'effet du cycle économique sur le taux d'intérêt des crédits par le biais de son impact sur le niveau des prêts non performants $\left(\varphi_{2}^{A}\right)$ ainsi que sur celui des passations en charges $\left(\varphi_{2}^{B}\right)$. Plus précisément, suite à une modification du cycle économique, les prêts non performants augmentent dans une proportion $\omega$, ce qui conduit la banque à percevoir moins d'intérêts $\left(r^{L} j_{0}\right)$ et à constituer davantage de provisions $\left(Z h_{0} j_{0}\right)$. De plus, suite à une modification du cycle économique, les passations en charges augmentent dans une proportion $\theta$, ce qui conduit la banque à percevoir moins d'intérêt $\left(r^{L} g_{0}\right)$ et à subir une perte non anticipée $\left(Z \delta g_{0}\right)$. Les modifications des réserves pour pertes $\left(L \hat{L} R_{t}\right)$, résultant des modifications du cycle économique, affectent également le comportement de la banque dans la mesure où elles déterminent la valeur net du portefeuille de crédit et donc le montant de dettes contracté par la banque.

L'équation (13) permet ainsi de mettre en évidence que les règles de provisionnement en vigueur affectent le comportement de la banque sur le marché du crédit. Dans le cadre d'un système de provisionnement ex post, les provisions constituées par les banques dépendent des évolutions des prêts non performants. Cette pratique de provisionnement amplifie donc l'effet du cycle économique $\left(\hat{y}_{t}\right)$ sur le marché du crédit par rapport à une situation où le coût de provisionnement supporté par la banque ne varirait pas au cours du cycle économique. Cette amplification est représentée par la présence des termes $\omega Z h_{0} j_{0}$ dans le coefficient $\varphi_{2}$ et $-\omega Z h_{0} j_{0} \beta r^{M}$ dans le coefficient $\varphi_{3}$. Plus exactement, l'effet marginal du cycle économique sur le taux des crédits via la régle de provisionnement ex 
post est donné par $\omega Z h_{0} j_{0}\left(1-\beta r^{M}\right) / \varphi_{0}$. Par conséquent, les effets sur le marché du crédit de tout type de choc affectant l'activité économique sont amplifiés. Nous appellons cet effet le canal des provisions bancaires.

\subsection{Solutions pour atténuer le canal des provisions bancaires}

Plusieurs solutions peuvent être envisagées pour atténuer le canal des provisons bancaires mis en évidence lorsqu'un système de provisionnement ex post est en vigueur. D'un point de vue réglementaire, une première solution consiste en l'adoption d'un système de provisionnement dynamique (ou ex ante) dans lequel les banques doivent lisser leurs provisions pour pertes dans le temps. Au niveau de la banque, la constitution d'un "coussin de sécurité" en fonds propres représente une solution alternative permettant de compenser les effets liés aux évolutions contracycliques des provisions constituées par les banques.

\subsubsection{Mise en place d'un système de provisionnement dynamique}

Dans le système de provisionnement dynamique (ou statistique), les autorités de réglementation exigent que les banques constituent des provisions statistiques, en plus des provisions spécifiques, afin de compenser les évolutions contracycliques de provisions spécifiques (Fernandez de Lis et al., 2001). Les provisions totales sont donc lissées dans le temps et les réserves pour pertes couvrent les pertes potentielles de l'ensemble du portefeuille de crédits. Les provisions pour pertes dans le système de provisionnement dynamique sont ainsi définies par :

$$
L L P_{i, t}=\mathrm{H}^{\mathrm{SS}}\left(L_{i, t}\right)=h_{0} L_{i, t} j_{0}
$$


où $\mathrm{H}^{\mathrm{SS}}\left(L_{i, t}\right)$ représente la somme des provisions spécifiques et des provisions statistiques ${ }^{11}$. Les provisions totales sont lissées autour de la valeur à l'état stationnaire des pertes potentielles par période $\left(h_{0} L_{i, t} j_{0}\right)$. L'évolution contracyclique des prêts non performants résultant du cycle économique n'est plus un déterminant des provisions pour pertes. La dynamique du taux d'intérêt sur les crédits s'en trouve modifiée et est donnée par :

$$
\varphi_{0} \hat{r}_{t}^{L}=\varphi_{0} \beta \mathrm{E}_{t}\left\{\hat{r}_{t+1}^{L}\right\}+\varphi_{1}\left(\hat{r}_{t}^{M}-\beta \mathrm{E}_{t}\left\{\hat{r}_{t+1}^{M}\right\}\right)-\varphi_{2}^{\prime}\left(\hat{y}_{t}-\beta \mathrm{E}_{t}\left\{\hat{y}_{t+1}\right\}\right)+L \hat{L} R_{t}^{\prime}
$$

où $\varphi_{2}^{\prime} \equiv \omega r^{L} j_{0}+\varphi_{2}^{B}$ et $L \hat{L} R_{t}^{\prime} \equiv-\varphi_{3}^{\prime} \hat{y}_{t}$ avec $\varphi_{3}^{\prime} \equiv \varphi_{3}^{B}$.

L'effet amplificateur que les règles de provisionnement peuvent avoir dans un système ex post n'est plus présent. L'effet marginal du cycle économique sur le taux des crédits est donc plus faible $\left(\varphi_{2}^{\prime}+\varphi_{3}^{\prime}<\varphi_{2}+\varphi_{3}\right)$. La mise en place d'un système de provisionnement dynamique permet donc d'éliminer l'effet amplificateur du canal des provisions bancaires.

\subsubsection{Constitution d'un capital buffer}

Lorsque la réglementation en vigueur requière d'appliquer des règles de provisionnement strictement ex post, la banque peut prendre en compte les faiblesses et imperfections de ce système de provisionnement. Elle cherchera alors à parvenir à une meilleure couverture de ses pertes potentielles et donc à une meilleure estimation de son profit économique ${ }^{12}$. Pour ce faire, la banque va accumuler des fonds propres excédentaires $\left(K B_{i, t}\right)$ pour couvrir les pertes potentielles qui ne sont pas couvertes par les réserves pour pertes, c'est-à-dire

\footnotetext{
${ }^{11}$ Les provisions statistiques sont donc égales à $h_{0} L_{i, t} j_{0}\left(1-\left(y_{t} / y\right)^{-\omega}\right)$.

${ }^{12}$ En pratique, les banques détiennent plus de fonds propres que le minimum requis par la réglementation bancaire. Sur la période 1997-2004 au sein de l'Union européenne (à 15 pays), les fonds propres détenus par les banques dépassaient en moyenne de 2,93 points de pourcentage le minimum réglementaire (Jokipii et Milne, 2006). Plusieurs raisons ont été avancées pour expliquer pourquoi les banques détiennent des excédents de fonds propres réglementaires (Jokipii and Milne, 2006). Parmi ces raisons, l'excédent de fonds propres réglementaires peut servir à couvrir une partie des pertes attendues.
} 
les pertes attendues non avérées. La loi d'accumulation du capital excédentaire est alors définie par :

$$
K B_{i, t+1}=K B_{i, t}+\eta\left(\mathrm{H}^{\mathrm{SS}}\left(L_{i, t}\right)-\mathrm{H}^{\mathrm{S}}\left(L_{i, t}, y_{t}\right)\right)
$$

Le montant $\left(\mathrm{H}^{\mathrm{SS}}\left(L_{i, t}\right)-\mathrm{H}^{\mathrm{S}}\left(L_{i, t}, y_{t}\right)\right)$ représente la différence entre les provisions pour pertes dans le système de provisionnement dynamique et les provisions pour pertes dans le système de provisionnement ex post, c'est-à-dire les provisions statistiques. Une fraction $\eta \in[0 ; 1]$, fixée de façon exogène, est ajoutée aux fonds propres excédentaires déjà existants. Les fonds propres excédentaires sont ajoutés aux fonds propres standards $\left(K_{i, t}\right)$. Nous définissons donc le total des fonds propres $\left(T K_{i, t}\right)$ par :

$$
T K_{i, t}=K_{i, t}+K B_{i, t}
$$

Nous supposons que le capital buffer est investi dans un actif sans risque $\left(S_{i, t}\right)$ rémunéré au taux $r_{t}^{M}$. Le bilan de la banque devient donc :

$$
L_{i, t}+S_{i, t}-L L R_{i, t}=D_{i, t}+T K_{i, t}
$$

De plus, l'origine et la répartition des profits de la banque sont données par :

$$
T K_{i, t+1}-T K_{i, t}+\Delta_{i, t}=r_{i, t}^{L} L_{i, t}\left(1-\mathrm{J}\left(y_{t}\right)-\mathrm{G}\left(y_{t}\right)\right)+r_{t}^{M} S_{i, t}-r_{t}^{M} D_{i, t}-L L P_{i, t}-\delta L_{i, t} \mathrm{G}\left(y_{t}\right) .
$$

La dynamique du taux d'intérêt sur les crédits est modifiée et est définie par :

$$
\varphi_{0} \hat{r}_{t}^{L}=\varphi_{0} \beta \mathrm{E}_{t}\left\{\hat{r}_{t+1}^{L}\right\}+\varphi_{1}\left(\hat{r}_{t}^{M}-\beta \mathrm{E}_{t}\left\{\hat{r}_{t+1}^{M}\right\}\right)-\varphi_{2}^{\prime \prime}\left(\hat{y}_{t}-\beta \mathrm{E}_{t}\left\{\hat{y}_{t+1}\right\}\right)+L \hat{L} R_{t}^{\prime \prime}
$$


où $\varphi_{2}^{\prime \prime} \equiv \omega\left(r^{L} j_{0}+Z(1-\eta) h_{0} j_{0}\right)+\varphi_{2}^{B}$ et $L \hat{L} R_{t}^{\prime \prime} \equiv-\varphi_{3}^{\prime \prime} \hat{y}_{t}$ avec $\varphi_{3}^{\prime \prime} \equiv(1-\eta) \varphi_{3}^{A}+\varphi_{3}^{B}$.

Si le paramètre $\eta$ est égal à 1 , les fonds propres excédentaires et les réserves pour pertes sont combinés afin de parvenir à une couverture complète des pertes potentielles. Ainsi, le comportement de la banque se trouve identique à celui observé dans le cadre d'un système de provisionnement dynamique $\left(\varphi_{2}^{\prime \prime}=\varphi_{2}^{\prime}\right.$ et $\left.\varphi_{3}^{\prime \prime}=\varphi_{3}^{\prime}\right)$. Les règles de provisionnement n'amplifient donc pas les effets du cycle économique. Cependant, le respect de cette contrainte $(\eta=1)$ peut conduire, pendant la phase haute du cycle économique, à une accumulation importante de fonds propres excédentaires qui seront utilisés pendant la phase basse du cycle. Les actionnaires pourraient ne pas apprécier un tel comportement et demander des dividendes plus élevés (impliquant $\eta<1$ ).

\section{Simulations}

Les effets des règles de provisionnement sur les fluctuations du marché du crédit sont illustrés à l'aide de simulations. Le modèle développé précédemment définit uniquement la dynamique du taux d'intérêt des crédits dans le cadre de différents systèmes de provisionnement. Des équations supplémentaires doivent donc être ajoutées de façon à compléter le modèle. Plus précisément, nous définissons également les dynamiques de la production $\left(\hat{y}_{t}\right)$, du taux d'intérêt sur les dettes bancaires $\left(\hat{r}_{t}^{M}\right)$, du taux d'inflation $\left(\hat{\pi}_{t}\right)$ et de la demande agrégée de crédits $\left(\hat{L}_{t}\right)$. La production est représentée par une relation IS. Le taux d'intérêt sur les dettes bancaires est supposé égal au taux du marché monétaire. Nous considérons que ce dernier est déterminé par une règle de Taylor. Le taux d'inflation est modélisé par une relation de Phillips. Enfin, la spécification de la demande agrégée de crédit se fonde sur les travaux de Calza et al. (2006). Nous complétons donc notre 
modèle avec les équations suivantes :

$$
\begin{gathered}
\hat{y}_{t}=\left(1-\alpha_{y}\right) \hat{y}_{t-1}+\alpha_{y} \mathrm{E}_{t}\left\{\hat{y}_{t+1}\right\}-\alpha_{r^{M}}\left(\hat{r}_{t}^{M}-\mathrm{E}_{t}\left\{\hat{\pi}_{t+1}\right\}\right), \\
\hat{\pi}_{t}=\left(1-\gamma_{\pi}\right) \hat{\pi}_{t-1}+\gamma_{\pi} \mathrm{E}_{t}\left\{\hat{\pi}_{t+1}\right\}+\gamma_{y} \hat{y}_{t}, \\
\hat{r}_{t}^{M}=\rho_{r^{M}} \hat{r}_{t-1}^{M}+\left(1-\rho_{r^{M}}\right)\left(\psi_{\pi} \mathrm{E}_{t}\left\{\hat{\pi}_{t+1}\right\}+\psi_{y} \hat{y}_{t}\right)+\hat{\varepsilon}_{t}, \\
\hat{L}_{t}=\phi_{y} \hat{y}_{t}-\phi_{r^{L}}\left(\hat{r}_{t}^{L}-\mathrm{E}_{t}\left\{\hat{\pi}_{t+1}\right\}\right),
\end{gathered}
$$

où $\hat{\varepsilon}_{t}$ est un choc de politique monétaire de type bruit blanc. Nous pouvons remarquer que, compte tenu des spécifications retenues, les dynamiques de la production, de l'inflation et du taux d'intérêt du marché monétaire ne dépendent pas des conditions du marché du crédit. Les simulations réalisées cherchent ainsi à illustrer l'ampleur des fluctations sur le marché du crédit en fonction de la réglementation sur les provisions pour pertes. Elles ne cherchent pas à illustrer l'effet des conditions du marché du crédit sur les fluctuations des principales variables macroéconomiques.

\subsection{Calibrage}

Nous cherchons à adapter notre calibration à la situation de l'Union économique et monétaire (UEM).

\subsubsection{L'équation du taux d'intérêt des crédits}

La dynamique du taux d'intérêt sur les crédits est donnée par l'équation (13), (15) ou (20) selon la spécification du modèle considéré.

Les valeurs à l'état stationnaire du taux des crédits $\left(r^{L}\right)$ et du taux du marché moné- 
taire $\left(r^{M}\right)$ sont calibrées à partir de données marcroéconomiques. Le taux des crédits pour l'UEM, fourni par Henzel et al. (2007), est disponible pour la période 1991Q12002Q4. Sa valeur moyenne sur cette période est de 8,5\%. Le modèle est calibré sur une base trimestrielle. La valeur à l'état stationnaire du taux des crédits $\left(r^{L}\right)$ est donc fixée à 2,1\%. De façon équivalente, la valeur du paramètre $r^{M}$ est calibrée en utilisant les données du taux du marché monétaire disponibles dans la base Area-Wide Model (Fagan et al., 2005). La moyenne du taux du marché monétaire sur la période 1991Q1-2002Q4 est de $6,2 \%$, ce qui correspond en base trimestrielle à un taux de $1,5 \%$.

Nous utilisons un panel non cylindré de 1249 banques de l'UEM ${ }^{13}$ sur la période 19922005 pour calibrer les paramètres portant sur les prêts non performants. Ce panel est construit à partir de la base de données Bankscope Fitch IBCA. La proportion de prêts non performants $\left(j_{0}\right)$ est mesurée par la valeur moyenne du ratio entre le montant de prêts non performants et le montant total des prêts accordés par la banque. Ce ratio est évalué à $7 \%$ sur notre panel. Par ailleurs, nous utilisons le ratio entre les provisions pour pertes et les prêts non performants pour calibrer le paramètre $h_{0}$. La valeur moyenne de ce ratio est de $20 \%$ sur notre panel. Chaque année, les provisions permettent donc de couvrir $20 \%$ de la valeur des prêts non performants, ce qui correspond en base trimestrielle à un taux de couverture de $4,7 \%$. Nous devons également calibrer le paramètre $\omega$ représentant l'élasticité des prêts non performants à l'output gap. Nous sommes ainsi amenés à estimer une équation sur les déterminants du ratio entre les prêts non performants et le total des prêts. Nous considérons comme variables explicatives la valeur retardée de ce ratio et l'output gap $^{14}$. Les coefficients associés à ces deux variables explicatives ont un effet

\footnotetext{
${ }^{13}$ Nous considérons des banques commerciales, coopératives et mutualistes et des caisses d'épargne. Au total, nous avons 7 banques d'Allemagne, 7 d'Autriche, 1 de Belgique, 83 d'Espagne, 9 de Finlande, 269 de France, 6 de Grèce, 832 d'Italie, 7 des Pays Bas et 28 du Portugal.

${ }^{14}$ Nous estimons l'équation : $N P L_{i, t}=c+\rho_{1} N P L_{i, t-1}+\lambda_{1} \hat{y}_{i, t}+e_{i, t}$ où $N P L_{i, t}$ représente le ratio des prêts non performants et $\lambda_{1}=\left(1-\rho_{1}\right) \omega$. Les résultats de ces estimation sont disponibles auprès des
} 
significatif. Le coefficient de long terme associé à l'output gap est estimé à 2,10 et est utilisé pour calibrer $\omega$.

Dans la base de données Bankscope Fitch IBCA, un nombre limité de banques fournissent des informations concernant leurs passations en charges. Nous considérons donc un panel de banques européennes et non plus seulement de l'UEM pour calibrer les paramètres portant sur les passations en charges. Nous obtenons ainsi de l'information sur les passations en charges pour un panel non cylindré de 628 banques $^{15}$ sur la période 1992-2005. Le ratio entre les passations en charge et le total des prêts est utilisé pour calibrer la proportion des prêts passés en charges $g_{0}$. La valeur moyenne de ce ratio est de 1,4\%. Pour une base trimestrielle, le paramètre $g_{0}$ est donc fixé à $0,36 \%$. Par ailleurs, nous estimons une équation sur les déterminants du ratio entre les prêts passés en charges et le total des prêts pour calibrer l'élacticité des passations en charges à l'output gap $(\theta)$. Les variables explicatives considérées sont la valeur retardée de la variable endogène et l'output gap ${ }^{16}$. Le coefficient de long terme associé à l'output gap est estimé à 0,22 et est utilisé pour calibrer le paramètre $\theta$.

La contrainte réglementaire sur les fonds propres, $k_{0}$, est fixée à $4 \%$ correspondant à la valeur du Tiers 1 . Le facteur d'actualisation est calibrée à 0,99. Cette valeur est généralement retenue dans la littérature et correspond à un taux d'intérêt en base annuelle à l'état stationnaire de $4 \%$. Le paramètre $\eta$ dans le système de capital buffer est fixé à 1 afin de fermer le canal des provisions bancaires.

Les paramètres $\delta$ and $\zeta$ sont déduits de l'état stationnaire du modèle. L'équation (7) montre qu'à l'état stationnaire, les provisions pour pertes sont égales aux passations en

\footnotetext{
auteurs.

${ }^{15}$ Notre panel se compose de 9 banques d'Allemagne, 3 d'Autriche, 64 d'Espagne, 7 de France, 6 de Grèce, 406 d'Italie, 26 de Norvège, 5 des Pays Bas, 18 du Portugal et 84 du Royaume-Uni.

${ }^{16}$ Nous estimons l'équation : $C O_{i, t}=c^{\prime}+\rho_{2} C O_{i, t-1}+\lambda_{2} \hat{y}_{i, t}+e_{i, t}^{\prime}$ où $C O_{i, t}$ est le ratio des passations en charges et $\lambda_{2}=\left(1-\rho_{2}\right) \theta$. Les résultats de ces estimations sont disponibles auprès des auteurs.
} 
Table 1: Model calibration

\begin{tabular}{|c|c|c|}
\hline Paramètre & Notation & Calibrage \\
\hline \multicolumn{3}{|l|}{$\triangleright$ Equation sur le taux des crédits } \\
\hline Taux sur les crédits à l'état stationnaire & $r^{L}$ & $2,10 \%$ \\
\hline Taux du marché monétaire à l'état stationnaire & $r^{M}$ & $1,50 \%$ \\
\hline Proportion des prêts non performants & $j_{0}$ & $7,00 \%$ \\
\hline Proportion des passations en charges & $g_{0}$ & $0,36 \%$ \\
\hline Proportion de provisions pour pertes & $h_{0}$ & $4,70 \%$ \\
\hline Contrainte réglementaire sur les fonds propres & $k_{0}$ & $4,00 \%$ \\
\hline Proportion de passations en charges anticipées & $\delta$ & $8,60 \%$ \\
\hline Elacticité des prêts non performants à l'output gap & $\omega$ & 2,10 \\
\hline Elacticité des passations en charges à l'output gap & $\theta$ & 0,22 \\
\hline Mark-up & $\zeta$ & 21 \\
\hline Facteur d'actualisation & $\beta$ & 0,99 \\
\hline Capital buffer & $\eta$ & 1 \\
\hline \multicolumn{3}{|l|}{$\triangleright$ Relation IS (équation (21)) } \\
\hline Poids de la composante forward looking & $\alpha_{y}$ & 0,50 \\
\hline Elasticité au taux du marché monétaire & $\alpha_{r^{M}}$ & 0,06 \\
\hline \multicolumn{3}{|l|}{$\triangleright$ Relation de Phillips (équation (22)) } \\
\hline Poids de la composante forward looking & $\gamma_{\pi}$ & 0,50 \\
\hline Elasticité au coût marginal réel & $\gamma_{y}$ & 0,01 \\
\hline \multicolumn{3}{|l|}{$\triangleright$ Règle de Taylor (équation (23)) } \\
\hline Comportement de lissage & $\rho_{r^{M}}$ & 0,70 \\
\hline Réaction à l'inflation anticipée & $\psi_{\pi}$ & 1,75 \\
\hline Réaction à l'output gap & $\psi_{y}$ & 0,60 \\
\hline Autocorrélation du choc de politique monétaire & $\rho_{\varepsilon}$ & 0,25 \\
\hline \multicolumn{3}{|l|}{$\triangleright$ Demande de crédit agrégée (équation (24)) } \\
\hline Elasticité à l'output gap & $\phi_{y}$ & 1,48 \\
\hline Elasticité au taux sur les crédits & $\phi_{r^{L}}^{g}$ & 5,08 \\
\hline
\end{tabular}


charges anticipées : $h_{0} j_{0} L=(1-\delta) g_{0} L$. La part non anticipée des passations en charges est ainsi définie par : $\delta=1-h_{0} j_{0} / g_{0}=0,086$. Par ailleurs, l'équation (25) montre que le mark-up est donné par : $\frac{\zeta}{\zeta-1}=\frac{r^{L}\left(1-j_{0}-g_{0}\right)}{r^{M}\left(1-k_{0}\right)+\delta g_{0}+h_{0} j_{0}+\left(k_{0} / \beta-k_{0}\right) /(1-\tau)}=1,05$. Nous obtenons ainsi un mark-up de $5 \%$ ce qui correspond à une élasticité de substitution $\zeta$ égale à 21 .

\subsubsection{Les équations complémentaires}

Le poid affecté à la composante forward looking respectivement dans la relation IS $\left(\alpha_{y}\right)$ et dans la relation de Phillips $\left(\gamma_{\pi}\right)$ est de 0,5. Ce calibrage se base sur les résultats de Sahuc (2002) qui estime un modèle nouveau keynésien standard pour l'UEM. Dans la relation IS, l'élasticité au taux d'intéret réel du marché monétaire $\left(\alpha_{r^{M}}\right)$ est fixée à 0,6 d'après les résultats de Sahuc (2002). Dans la relation de Phillips, l'élasticité au coût marginal réel $\left(\gamma_{y}\right)$ est calibrée à 0,01 d'après les estimations réalisées par Smets et Wouters (2003).

Le calibrage de la règle de Taylor se base sur les travaux de Gerdesmeier et Roffia (2004), Mésonnier et Renne (2004), Hayo et Hofmann (2006) et Fendel et Frenkel (2006). Ces travaux estiment des fonctions de réaction pour la Banque Centrale Européenne $(\mathrm{BCE})$ et montrent que la réponse à l'inflation anticipée, réprésentée par le coefficient $\psi_{\pi}$, est comprise entre 1,5 et 2. De plus, la réponse à la production, représentée par le coefficient $\psi_{y}$, est comprise entre 0,50 et 0,75 . Nous calibrons donc les paramètres $\psi_{\pi}$ et $\psi_{y}$ respectivement à 1,75 à 0,60 . Par ailleurs, ces travaux montrent que le comportement de lissage de la $\mathrm{BCE}$, mesuré par le paramètre $\rho_{r^{M}}$, est élevé (entre 0,80 et 0,95 ). Nous calibrons le paramètre $\rho_{r^{M}}$ à 0,85 .

Les paramètres de la relation représentant la demande agrégée de crédits sont calibrés d'après les estimations de Calza et al. (2006). Ces auteurs obtiennent pour l'UEM une élasticité à la production $\left(\phi_{y}\right)$ égale à 1,48 et une élasticité au taux d'intérêt réel sur les 
crédits $\left(\phi_{r^{L}}\right)$ de 5,08 .

\subsection{Fonctions de réponse}

Nous analysons les fonctions de réponse à un choc de politique monétaire $\left(\hat{\varepsilon}_{t}\right)^{17}$. Le choc considéré est normalisé à $1 \%$ et nous représentons les fonctions de réponse sur 20 périodes. Nous considérons deux spécifications pour le modèle. Dans la première spécification, le canal des provisions bancaires est actif, c'est-à-dire que les banques appliquent des règles de provisionnement ex post. Dans la seconde spécification, le canal des provisions bancaires est inactif. Des règles de provisionnement ex ante ou l'utilisation d'un capital buffer permettent de fermer le canal des provisions bancaires. De même, nous pourrions considérer des situations intermédiaires dans lesquelles le système de capital buffer permet de fermer partiellement le canal des provisions bancaires.

Les fonctions de réponse des variables du modèle à un choc de politique monétaire $\left(\varepsilon_{r, t}\right)$ sont représentées sur le graphique 1. Les effets de ce choc sur le taux du marché monétaire, la production et l'inflation sont identiques dans les deux spécifications du modèle dans la mesure où les conditions sur le marché du crédit n'affectent pas ces variables. L'augmentation immédiate du taux du marché monétaire provoquée par le choc détériore la demande agrégée, ce qui se traduit par un effet récessif sur la production et un ralentissement de l'inflation ${ }^{18}$. Les différences entre les deux spécifications du modèle se manifestent au niveau de la réaction instantanée du marché du crédit au choc

\footnotetext{
${ }^{17}$ Nous nous focalisons sur la transmission des effets de la politique monétaire sur le marché du crédit mais les conclusions auxquelles nous parvenons ne sont pas liées spécifiquement à ce type de choc. Nous aurions pu, par exemple, considérer un choc de demande, un choc d'offre ou encore un choc sur les prêts non performants.

${ }^{18}$ Nous pouvons remarquer qu'en pratique, les effets d'un choc de politique monétaire ne se manifestent pas immédiatement sur la production et l'inflation. Pour reproduire ce décalage des effets d'un choc de politique monétaire, il faudrait supposer que la production et l'inflation soient des variables prédéterminées, c'est-à-dire qu'elles soient déterminées avant l'occurrence du choc. Les fonctions de réponses à un choc de politique monétaire obtenues quand la production et l'inflation sont prédéterminées sont disponibles auprès des auteurs. Les conclusions auxquelles nous parvenons ne sont pas modifiées.
} 
de politique monétaire. Quand le canal des provisions bancaires est inactif, la réponse positive du taux des crédits s'explique principalement par la répercussion de la hausse du taux du marché monétaire. Dans le système de provisionnement ex post, un effet supplémentaire se manifeste. La contraction de la production provoquée par le choc de politique monétaire s'accompagne d'une augmentation des prêts non performants et donc des provisions pour pertes effectuées par les banques. Cette hausse des coûts supportés par les banques est répercutée sur le taux des crédits. Ainsi, la réponse instantanée du taux des crédits est de 0,78\% quand le canal des provisions bancaires est inactif et de 0,89\% quand il est actif. Par conséquent, la contraction des crédits est également plus prononcée quand les banques appliquent des règles de provisionnement ex post. L'effet récessif du choc de politique monétaire sur la production perdure une dizaine de périodes. Les conditions sur le marché du crédit demeurent donc plus restrictives dans le système de provisionnement ex post que dans un système dans lequel le canal des provisions bancaires est inactif pendant cette dizaine de périodes.

\subsection{Analyse de la sensibilité}

L'équation (15) montre que l'élasticité du taux des crédits à la production est donnée par $-\left(\varphi_{4}^{\prime}+\varphi_{7}^{\prime}\right) / \varphi_{0}=-0,16$ quand le canal des provisions bancaires est inactif. Le canal des provisions bancaires apparaît à travers une amplification de cette élasticité. Cette amplification est donnée par $-\left[\left(\varphi_{4}-\varphi_{4}^{\prime}\right)+\left(\varphi_{7}-\varphi_{7}^{\prime}\right)\right] / \varphi_{0}=-0,37$. Le graphique 2-a illustre le rôle des prêts non performants dans cette amplification. Le canal des provisions bancaires dépend positivement de la proportion des prêts non performants $\left(j_{0}\right)$ et de l'élasticité des prêts non performants à l'output gap $(\omega)$. Le graphique 2-a montre que ces deux paramètres sont complémentaires. Un canal des provisions bancaires 
Figure 1: Fonctions de réponse sur 20 periodes à un choc de politique monétaire

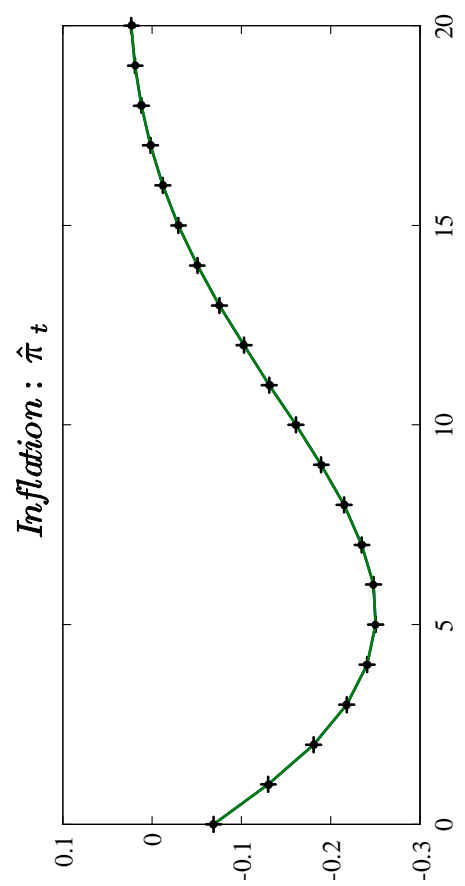

(\%) иоџฺчиәр

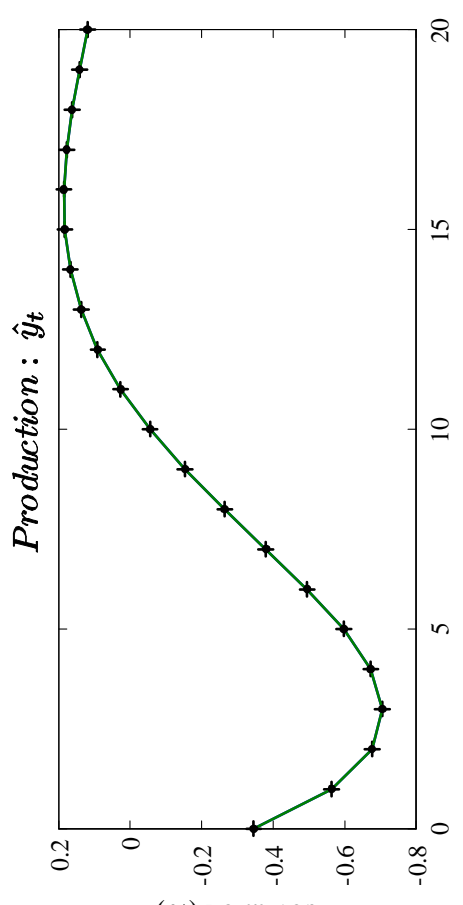

(\%) иоџฺчцәр

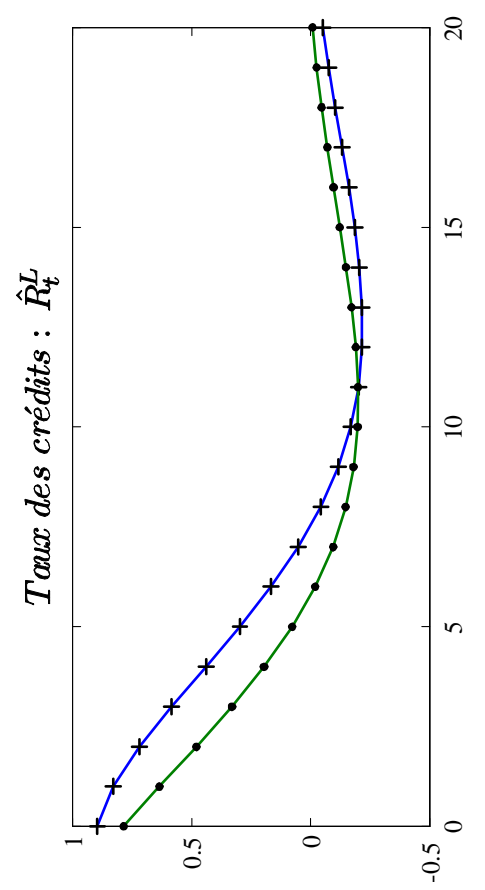

(\%) чоџฺฺ

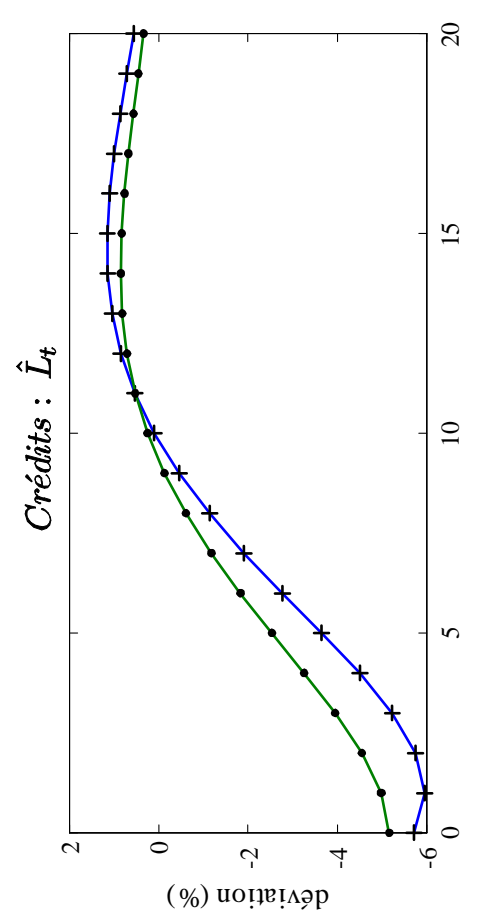

(\%) иоџฺ เฺрр

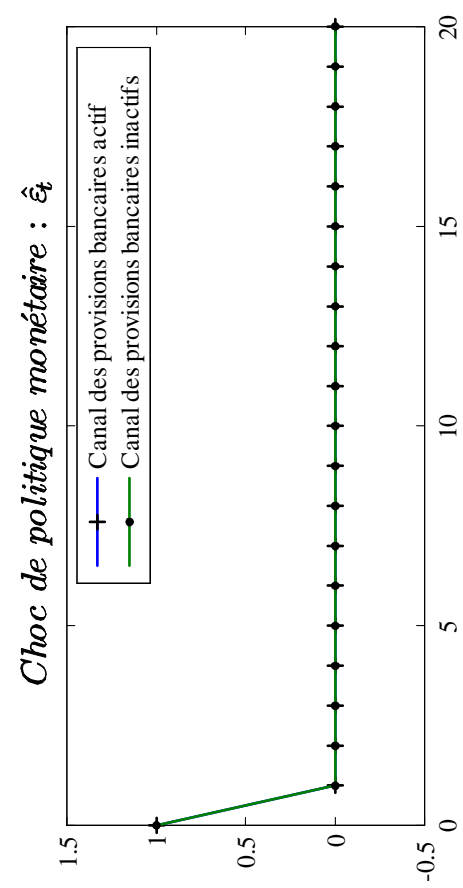

(\%) иоџฺฺ $\Lambda ә$

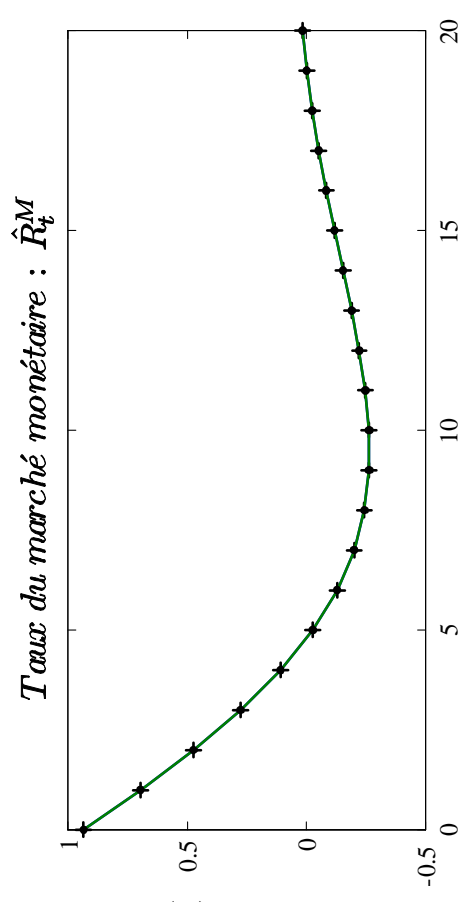

(\%) иоџฺ̣ 
Figure 2: Déterminants du canal des provisions bancaires

a- Prêts non performants

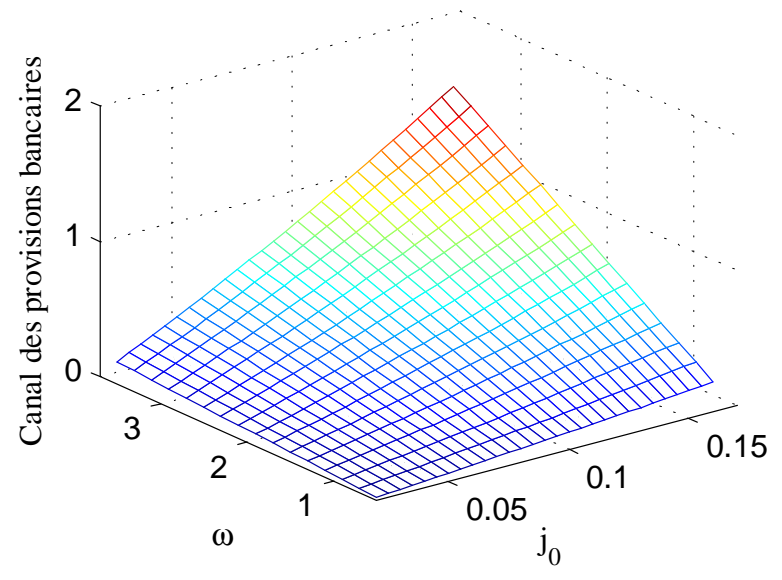

b- Provisions pour pertes et mark-up

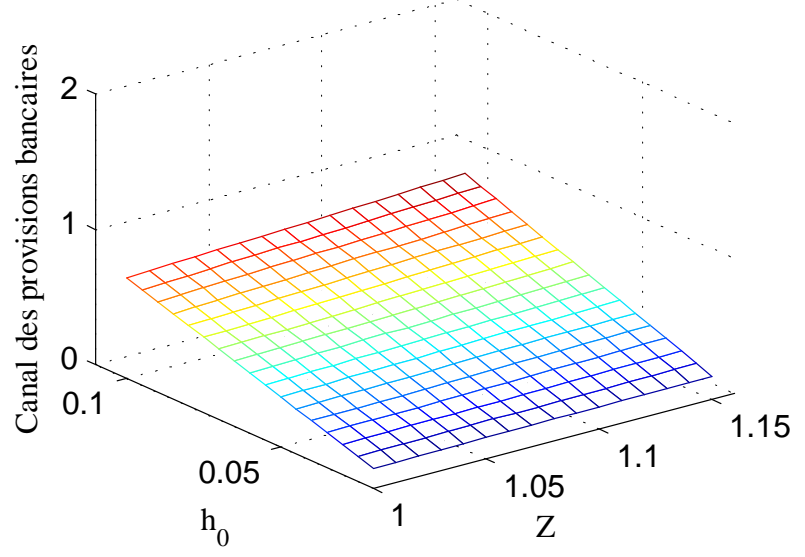

important requiert à la fois que les banques fassent face en moyenne à des prêts non performants relativement conséquents et que ces prêts non performants soient marqués par des évolutions procycliques. Le graphique 2-a indique également que le canal des provisions bancaires pourrait varier sensiblement entre les banques en fonction de leurs performances en matière de prêts non performants.

La proportion de provisions pour pertes constituées par les banques $\left(h_{0}\right)$ et le mark-up $(Z \equiv \zeta /(\zeta-1))$ contribuent également positivement au canal des provisions bancaires. Le graphique 2-b montre cependant que l'intensité du canal des provisions bancaires est sensible uniquement aux modifications du paramètre $h_{0}$. Ce dernier correspond au ratio entre les provisions pour pertes et les prêts non performants. Il est ainsi associé à la vitesse à laquelle les banques couvrent leurs prêts non performants. Le canal des provisions bancaires est donc plus important quand les banques n'ont pas la possibilité de conserver leurs prêts classés comme non performants durant une période suffisament longue pour constituer progressivement leurs provisions pour pertes.

Le graphique 3 illustre la sensibilité des fonctions de réponse à un choc de poli- 
tique monétaire quand la calibration d'un paramètre est modifiée toutes choses égales par ailleurs. Plus précisément, nous considérons alternativement une modification de la proportion des prêts non performants $\left(j_{0}\right)$, de la proportion moyenne des prêts non performants provisionnés par période $\left(h_{0}\right)$ et de la réaction à la production de la banque centrale $\left(\psi_{y}\right)$.

Premièrement, nous envisageons un doublement de la proportion des prêts non performants. Le paramètre $j_{0}$ est donc calibré à $14 \%$. L'amplification de l'élasticité du taux des crédits à la production résultant du canal des provisions bancaires passe ainsi à $-0,79$ contre $-0,37$ dans le scénario de référence ${ }^{19}$. Les effets du choc de politique monétaire sur le marché du crédit deviennent donc plus importants. Le ralentissement économique provoqué par le choc est accompagné par davantages de prêts non performants, la réponse instantanée du taux des crédits au choc est donc de 1,14\% alors qu'elle était de 0,89\% avec le calibrage de référence. La contraction du montant de crédits distribués suivant le choc est donc plus prononcée. Les conditions sur le marché du crédit demeurent plus restrictives sur une dizaine de périodes correspondant à la phase pendant laquelle le choc à un effet négatif sur la production.

La seconde modification envisagée porte sur la proportion moyenne des prêts non performants provisionnés par période. Le paramètre $h_{0}$ est calibré à 0,094 ce qui correspond à une multiplication par deux de ce paramètre par rapport au calibrage de référence. Les banques doivent ainsi provisionner plus rapidement les prêts non performants résultant de la propagation des effets du choc. L'amplification de l'élasticité du taux des crédits à la production résultant du canal des provisions bancaires est de $-0,73$ et la réponse instantanée du taux des crédits au choc passe à $1 \%$. De façon identique au scénario

\footnotetext{
${ }^{19}$ Cette amplification aurait pu également être obtenue en modifiant le paramètre $\omega$ comme nous l'avons remarqué à partir du graphique 2-a.
} 
précédent, les conditions sur le marché du crédit demeurent plus restrictives durant une dizaine de périodes.

La troisième modification envisagée porte sur la réaction de la banque centrale aux modifications de la production. Le paramètre $\psi_{y}$ est multiplié par deux et ainsi calibré à 1,2. Cette plus forte réactivité de la banque centrale permet de limiter la contration de la production suite au choc et donc de limiter la dégradation de la qualité du portefeuille de crédit de la banque. Cependant, la réaction du taux d'intérêt sur les crédits est relativement peu modifiée par rapport au calibrage de référence. La réponse instantanée de cette variable passe à $0,86 \%$ contre $0,89 \%$ dans le scénario de référence. Les caractéristiques du secteur bancaire (repésentées par exemple par les paramètres $j_{0}$ ou $h_{0}$ ) semblent donc plus importants que le comportement de la banque centrale pour expliquer l'ampleur de la réaction du marché du crédit au choc.

Figure 3: Sensibilité du canal des provisions bancaires
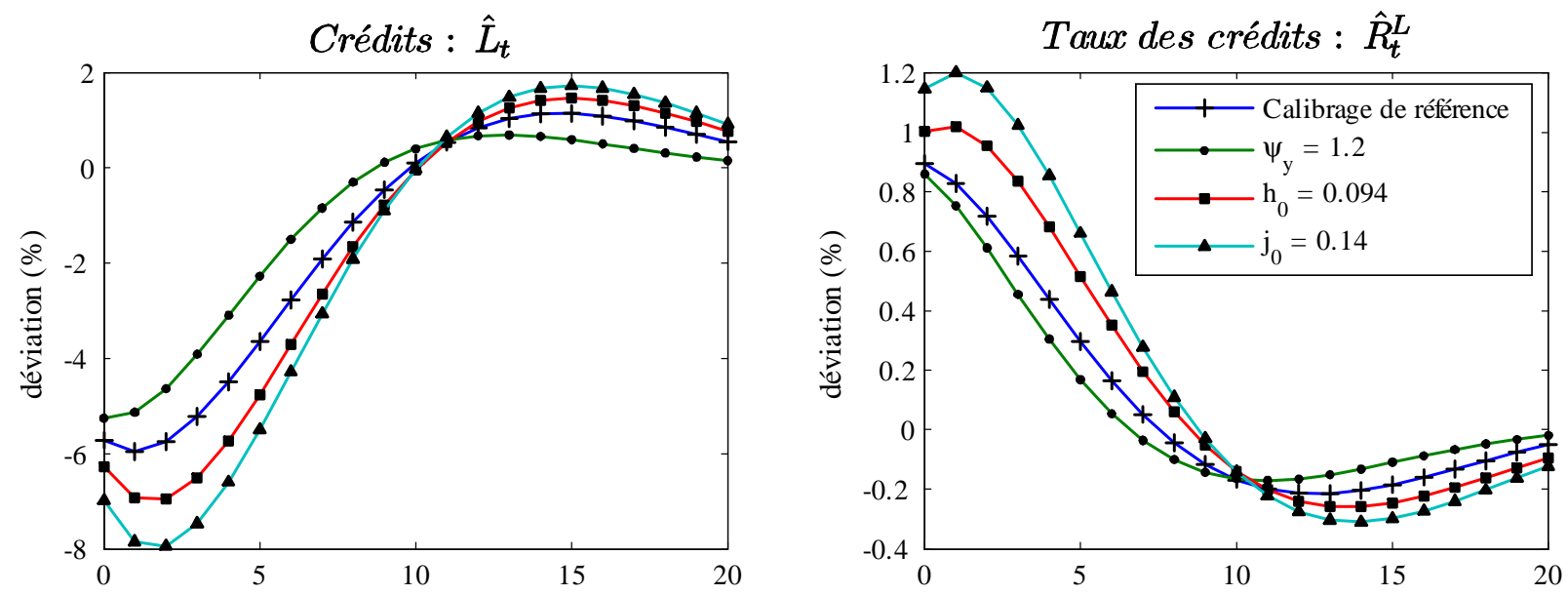


\section{Conclusion}

Cet article montre que la réglementation sur les provisions pour pertes peut contribuer à expliquer les fluctuations cycliques se produisant sur le marché du crédit. Plus précisément, le modèle développé et les simulations réalisées montrent qu'un système de provisionnement ex post amplifie les fluctuations de court terme sur le marché du crédit. Un tel effet n'est pas présent dans un système de provisionnement ex ante où la constitutions de provisions statistiques permet de lisser les provisions pour pertes à travers le cycle de l'activité économique. L'utilisation d'un capital buffer permet d'aboutir au même résultat mais implique une certaine variabilité dans les fonds propres détenus par les banques. Les conclusions de cet article sont donc favorables à la mise en oeuvre d'un système de provisionnement dynamique comparable à l'initiative prise par la Banque d'Espagne.

Plusieurs développements du modèle développé pourraient être envisagés. Par exemple, une littérature grandissante cherche à évaluer comment le comportement d'offre de crédit des banques est affecté par la réglementation sur les fonds propres (voir par exemple Furfine (2001), Van den Heuvel (2002) ou Zicchino (2006)). Il pourrait ainsi être intéressant de prendre en compte conjointement les effets de la réglementation sur les provisions pour pertes et ceux de la réglementation sur les fonds propres. Plus précisément, les montants de fonds propres détenus par les banques pourraient être plus stables dans le cadre d'un système de provisionnement dynamique ce qui réduirait les effets résultants de la contrainte réglementaire sur les fonds propres. Par ailleurs, une seconde extension du modèle pourrait consister à prendre en compte des rigidités sur le taux des crédits de façon à mieux rendre compte les délais d'ajustement du marché du crédit aux chocs. 


\section{References}

Arpa, M., Giulini, I., Ittner, A., Pauer, F., 2001. The influence of macroeconomic developments on Austrian banks: implications for banking supervision. BIS Papers, 1, 91-116.

Asea, P., Blomberg, B., 1998. Lending cycles. Journal of Econometrics, 83, 89-128.

Berger, A., Udell, G., 2004. The institutional memory hypothesis and the procyclicality of bank lending behavior. Journal of Financial Intermediation, 13, 458-495.

Bikker, J., Metzemakers, P., 2005. Bank provisioning behavior and procyclicality. Journal of International Financial Markets, Institutions and Money, 15, 141-157.

Borio, C., Furfine, C., Lowe, P., 2001. Procyclicality of the financial system and financial stability: issues and policy options. BIS Papers, 1, 1-57.

Calza, A., Manrique, M., Sousa, J., 2006. Credit in the euro area: An empirical investigation using aggregate data. The Quarterly Review of Economics and Finance, 46, 211-226.

Carletti, E., Hartmann, P., Spagnolo, G., 2007. Bank mergers, competition and liquidity. Journal of Money, Credit and Banking, 39, 1067-1105.

Clerc, L., Drumetz, F., Jaudoin, O., 2001. To what extent are prudential and accounting arrangements pro- or countercyclical with respect to overall financial conditions? BIS Papers, 1, 197-210.

Fagan, G., Henry, J., Mestre, R., 2005. An area-wide model (AWM) for the euro area. Economic Modelling, 22, 39-59.

Fendel, R., Frenkel, M., 2006. Five years of European single monetary policy: Is the ECB rule-based? Contemporary Economic Policy, 24, 106-115.

Fernandez de Lis, S., Martinez Pagès, J., Saurina, J., 2001. Credit growth, problem loans and credit risk provisioning in Spain. BIS Papers, 1, 331-353

Furfine, C., 2001. Bank portfolio allocation: the impact of capital requirements, regulatory monitoring and economic conditions. Journal of Financial Services Research, 20, 33-56.

Gerdesmeier, D., Roffia, B., 2004. Empirical estimates of reaction functions for the euro area. Swiss Journal of Economics and Statistics, 140, 37-66.

Guttentag, J., Herring, R., 1986. Disaster myopia in international banking. Princeton University, Essays in International Finance \#164.

Hayo, B., Hofmann, B., 2006. Comparing monetary policy reaction functions: ECB versus Bundesbank. Empirical Economics, 3, 645662.

Henzel, S., Hülsewig, O., Mayer, E., Wollmershäuser, T., 2007. The price puzzle revisited: can the cost channel explain a rise in inflation after a monetary policy shock? CESifo, Working Paper \#2039. 
Jiménez, G., Saurina, J., 2006. Credit cycles, credit risk, and prudential regulation. International Journal of Central Banking, 2, 65-98.

Jokipii, T., Milne, A., 2006. The cyclical behaviour of European bank capital buffers. Bank of Finland, Discussion Paper \#17/2006.

Laeven, L., Majnoni, G., 2003. Loan Loss Provisioning and Economic Slowdowns: Too Much, Too Late? Journal of Financial Intermediation, 12, 178-197.

Lown, C., Morgan, D., 2006. The credit cycle and the business cycle: new findings using the loan officer opinion survey. Journal of Money, Credit and Banking, 38, 1575-1598.

Mann, F., Michael, I., 2002. Dynamic provisioning: issues and application. Bank of England, Financial Stability Review, 12, 128-136.

Mésonnier, J.S., Renne, J.P., 2004. Règle de Taylor et politique monétaire dans la zone euro. Banque de France, NER \#117.

Pain, D., 2003. The provisioning experience of the major UK banks: A small panel investigation. Bank of England, Working Paper.\#177.

Rajan, R., 1994. Why bank credit policies fluctuate: A theory and some evidence. The Quaterly Journal of Economics, 109, 399-441.

Sahuc, J.G., 2002. A hybrid monetary policy model: Evidence from the Euro Area. Applied Economics Letters, 9, 949-955.

Van den Heuvel, S., 2002. The bank capital channel of monetary policy. Unpublished manuscript, University of Pennsylvania.

Walter, J., 1991. Loan loss reserves. Federal Reserve Bank of Richemond, Economic Review, July/August, 20-30.

Zicchino, L., 2006. A model of bank capital, lending and the macroeconomy: Basel I versus Basel II. Manchester School, Supplement 2006, 74, 50-77. 


\section{Appendice non destiné à publication}

\section{A. Le programme de maximisation}

\section{A.1 Le système de provisionnement ex post}

Le programme de maximisation dans le système de provisionnement ex post s'écrit :

$$
\max _{r_{i, t}^{L}} \mathrm{E}_{t} \sum_{j=0}^{\infty} \beta^{t+j} \Delta_{t+j}
$$

avec :

$$
\begin{aligned}
\Delta_{t}= & r_{i, t}^{L} L_{i, t}\left(1-j_{0}\left(y_{t} / y\right)^{-\omega}-g_{0}\left(y_{t} / y\right)^{-\theta}\right)-r_{t}^{M} D_{i, t} \\
& -h_{0} L_{i, t} j_{0}\left(y_{t} / y\right)^{-\omega}-\delta L_{i, t} g_{0}\left(y_{t} / y\right)^{-\theta}+k_{0} L_{i, t}-k_{0} L_{i, t+1},
\end{aligned}
$$

sous les contraintes :

$$
\begin{aligned}
D_{i, t} & =L_{i, t}-L L R_{i, t}-k_{0} L_{i, t}, \\
L L R_{i, t} & =L L R_{i, t-1}+h_{0} L_{i, t-1} j_{0}\left(y_{t-1} / y\right)^{-\omega}-(1-\delta) L_{i, t-1} g_{0}\left(y_{t-1} / y\right)^{-\theta},
\end{aligned}
$$

et :

$$
L_{i, t}=\left(\frac{r_{i, t}^{L}}{r_{t}^{L}}\right)^{-\zeta} L_{t} .
$$

La condition du premier ordre (CPO) sur le taux d'intérêt des crédits $\left(r_{i, t}^{L}\right)$ est donnée par :

$$
\begin{aligned}
r_{i, t}^{L}\left(1-\mathrm{J}\left(y_{t}\right)-\mathrm{G}\left(y_{t}\right)\right)= & \frac{\zeta}{\zeta-1}\left\{r_{t}^{M}\left(1-k_{0}\right)+\mathrm{E}_{t} \sum_{n=0}^{+\infty} \beta^{n+1} r_{t+1+n}^{M}\left[(1-\delta) \mathrm{G}\left(y_{t+n}\right)-h_{0} \mathrm{~J}\left(y_{t+n}\right)\right]\right. \\
& \left.+h_{0} \mathrm{~J}\left(y_{t}\right)+\delta \mathrm{G}\left(y_{t}\right)+\left(k_{0} / \beta-k_{0}\right)\right\} .
\end{aligned}
$$

\section{A.2 Le système de provisionnement ex ante}

Dans le système de provisionnement ex ante, l'équation (8) est remplacée par l'équation (14) dans le programme de maximisation. Les dividendes distribués aux actionnaires sont donc donnés par :

$$
\begin{aligned}
\Delta_{t}= & r_{i, t}^{L} L_{i, t}\left(1-j_{0}\left(y_{t} / y\right)^{-\omega}-g_{0}\left(y_{t} / y\right)^{-\theta}\right)-r_{t}^{M} D_{i, t} \\
& -\delta L_{i, t} g_{0}\left(y_{t} / y\right)^{-\theta}+k_{0} L_{i, t}-k_{0} L_{i, t+1}-h_{0} L_{i, t} j_{0},
\end{aligned}
$$

et la loi d'accumulation des réserves pour pertes est donnée par :

$$
L L R_{i, t}=L L R_{i, t-1}+h_{0} L_{i, t-1} j_{0}-(1-\delta) L_{i, t-1} g_{0}\left(y_{t-1} / y\right)^{-\theta} .
$$


La CPO sur le taux d'intérêt des crédits est donc donnée par :

$$
\begin{aligned}
r_{i, t}^{L}\left(1-\mathrm{J}\left(y_{t}\right)-\mathrm{G}\left(y_{t}\right)\right)= & \frac{\zeta}{\zeta-1}\left\{r_{t}^{M}\left(1-k_{0}\right)+\mathrm{E}_{t} \sum_{n=0}^{+\infty} \beta^{n+1} r_{t+1+n}^{M}\left[(1-\delta) \mathrm{G}\left(y_{t+n}\right)-h_{0} j_{0}\right]\right. \\
& \left.+\delta \mathrm{G}\left(y_{t}\right)+h_{0} j_{0}+\left(k_{0} / \beta-k_{0}\right)\right\} .
\end{aligned}
$$

\section{A.3 Le système de capital buffer}

Dans le système de capital buffer, les équations (16) et (17) sont prises en compte parmi les contraintes et l'équation (1) est remplacée par l'équation (18). De plus, les dividendes distribués aux actionnaires sont donnés par :

$$
\begin{aligned}
\Delta_{t}= & r_{i, t}^{L} L_{i, t}\left(1-j_{0}\left(y_{t} / y\right)^{-\omega}-g_{0}\left(y_{t} / y\right)^{-\theta}\right)-r_{t}^{M} D_{i, t} \\
& -\delta L_{i, t} g_{0}\left(y_{t} / y\right)^{-\theta}+k_{0} L_{i, t}-k_{0} L_{i, t+1} \\
& -(1-\eta) h_{0} L_{i, t} j_{0}\left(y_{t} / y\right)^{-\omega}-\eta h_{0} L_{i, t} j_{0}
\end{aligned}
$$

et la contrainte de bilan est donnée par :

$$
D_{i, t}=L_{i, t}+S_{i, t}-L L R_{i, t}-k_{0} L_{i, t}-K B_{i, t}
$$

avec

$$
L L R_{i, t}=L L R_{i, t-1}+h_{0} L_{i, t-1} j_{0}\left(y_{t-1} / y\right)^{-\omega}-(1-\delta) L_{i, t-1} g_{0}\left(y_{t-1} / y\right)^{-\theta},
$$

et

$$
S_{i, t}=K B_{i, t}=K B_{i, t-1}+\eta h_{0} L_{i, t-1} j_{0}\left(1-\left(y_{t-1} / y\right)^{-\omega}\right)
$$

La CPO sur le taux d'intérêt des crédits est donc donnée par :

$$
\begin{aligned}
r_{i, t}^{L}\left(1-\mathrm{J}\left(y_{t}\right)-\mathrm{G}\left(y_{t}\right)\right)= & \frac{\zeta}{\zeta-1}\left\{r_{t}^{M}\left(1-k_{0}\right)+\delta \mathrm{G}\left(y_{t}\right)\right. \\
& +\mathrm{E}_{t} \sum_{n=0}^{+\infty} \beta^{n+1} r_{t+1+n}^{M}\left[(1-\delta) \mathrm{G}\left(y_{t+n}\right)-\eta h_{0} j_{0}-h_{0}(1-\eta) \mathrm{J}\left(y_{t+n}\right)\right] \\
& \left.+h_{0}\left(\eta j_{0}+(1-\eta) \mathrm{J}\left(y_{t}\right)\right)+\left(k_{0} / \beta-k_{0}\right)\right\} .
\end{aligned}
$$

\section{B. La log-linéarisation}

A l'état stationnaire, la CPO sur le taux d'intérêt des crédits est identique pour les différentes spécifications du modèle. Elle est donnée par :

$$
r^{L}\left(1-j_{0}-g_{0}\right)=\frac{\zeta}{\zeta-1}\left\{r^{M}\left(1-k_{0}\right)+\delta g_{0}+h_{0} j_{0}+\left(k_{0} / \beta-k_{0}\right)\right\},
$$

où $r^{L}$ et $r^{M}$ représentent respectivement la valeur à l'état stationnaire du taux sur les crédits et du taux sur les dettes.

\section{B.1 Le système de provisionnement ex post}


La log-linéarisation de la CPO sur le taux des crédits dans le système de provisionnement ex post (équation (12))est donnée par :

$$
\begin{aligned}
\hat{r}_{t}^{L}\left[r^{L}\right]\left[1-j_{0}-g_{0}\right]= & \hat{r}_{t}^{M}\left[r^{M}\right]\left[(\zeta /(\zeta-1))\left(1-k_{0}\right)\right] \\
& +\hat{y}_{t}[y]\left[-r^{L}(1 / y)\left(\omega j_{0}+\theta g_{0}\right)-(\zeta /(\zeta-1))\left(\omega h_{0} j_{0}(1 / y)+\theta(1 / y) \delta g_{0}\right)\right] \\
& -\sum_{n=0}^{+\infty} \beta^{n+1} \mathrm{E}_{t}\left\{\hat{y}_{t+n}\right\}[y]\left[(\zeta /(\zeta-1)) r^{M}\left(\theta(1 / y)(1-\delta) g_{0}-\omega(1 / y) h_{0} j_{0}\right)\right],
\end{aligned}
$$

ce qui est équivalent à :

$$
\begin{aligned}
\hat{r}_{t}^{L}\left[r^{L}\left(1-j_{0}-g_{0}\right)\right]= & \hat{r}_{t}^{M}\left[r^{M} Z\left(1-k_{0}\right)\right] \\
& -\hat{y}_{t}\left[r^{L}\left(\omega j_{0}+\theta g_{0}\right)+Z\left(\omega h_{0} j_{0}+\theta \delta g_{0}\right)\right] \\
& -\sum_{n=0}^{+\infty} \beta^{n+1} \mathrm{E}_{t}\left\{\hat{y}_{t+n}\right\}\left[Z r^{M}\left(\theta(1-\delta) g_{0}-\omega h_{0} j_{0}\right)\right],
\end{aligned}
$$

avec $Z \equiv \zeta /(\zeta-1)$.

La log-linéarisation de l'équation (12) peut s'écrire :

$$
\varphi_{0} \hat{r}_{t}^{L}=\varphi_{1} \hat{r}_{t}^{M}-\varphi_{2} \hat{y}_{t}-\varphi_{3} \sum_{n=0}^{+\infty} \beta^{n} \mathrm{E}_{t}\left\{\hat{y}_{t+n}\right\}
$$

avec :

$$
\begin{aligned}
\varphi_{0} & \equiv r^{L}\left(1-j_{0}-g_{0}\right), \\
\varphi_{1} & \equiv Z r^{M}\left(1-k_{0}\right), \\
\varphi_{2}^{A} & \equiv r^{L} j_{0}+Z h_{0} j_{0}, \\
\varphi_{2}^{B} & \equiv r^{L} g_{0}+Z \delta g_{0}, \\
\varphi_{2} & \equiv \varphi_{2}^{A}+\varphi_{2}^{B}, \\
\varphi_{3}^{A} & \equiv-\omega\left(Z \beta r^{M} h_{0} j_{0}\right), \\
\varphi_{3}^{B} & \equiv \theta\left(Z \beta r^{M}(1-\delta) g_{0}\right), \\
\varphi_{3} & \equiv \varphi_{3}^{A}+\varphi_{3}^{B} .
\end{aligned}
$$

La transformation en quasi-différence $\left(\hat{r}_{t}^{L}-\beta \mathrm{E}_{t}\left\{\hat{r}_{t+1}^{L}\right\}\right)$ permet de simplifier la loglinéarisation de l'équation (12) et nous obtenons :

$$
\begin{aligned}
\varphi_{0} \hat{r}_{t}^{L}= & \varphi_{0} \beta \mathrm{E}_{t}\left\{\hat{r}_{t+1}^{L}\right\}+\varphi_{1}\left(\hat{r}_{t}^{M}-\beta \mathrm{E}_{t}\left\{\hat{r}_{t+1}^{M}\right\}\right) \\
& -\varphi_{2}\left(\hat{y}_{t}-\beta \mathrm{E}_{t}\left\{\hat{y}_{t+1}\right\}\right)-\varphi_{3} \hat{y}_{t} .
\end{aligned}
$$

Nous définissons $L \hat{L} R_{t} \equiv-\varphi_{3} \hat{y}_{t}$. Nous obtenons donc:

$$
\begin{aligned}
\varphi_{0} \hat{r}_{t}^{L}= & \varphi_{0} \beta \mathrm{E}_{t}\left\{\hat{r}_{t+1}^{L}\right\}+\varphi_{1}\left(\hat{r}_{t}^{M}-\beta \mathrm{E}_{t}\left\{\hat{r}_{t+1}^{M}\right\}\right) \\
& -\varphi_{2}\left(\hat{y}_{t}-\beta \mathrm{E}_{t}\left\{\hat{y}_{t+1}\right\}\right)+L \hat{L} R_{t} .
\end{aligned}
$$

\section{B.2 Le système de provisionnement ex ante}

La log-linéarisation de la CPO sur le taux des crédits dans le système de provisionnement 
ex ante est donnée par :

$$
\begin{aligned}
\hat{r}_{t}^{L}\left[r^{L}\right]\left[1-j_{0}-g_{0}\right]= & \hat{r}_{t}^{M}\left[r^{M}\right]\left[(\zeta /(\zeta-1))\left(1-k_{0}\right)\right] \\
& +\hat{y}_{t}[y]\left[-r^{L}(1 / y)\left(\omega j_{0}+\theta g_{0}\right)-(\zeta /(\zeta-1)) \theta(1 / y) \delta g_{0}\right] \\
& -\sum_{n=0}^{+\infty} \beta^{n+1} \mathrm{E}_{t}\left\{\hat{y}_{t+n}\right\}[y]\left[(\zeta /(\zeta-1)) r^{M} \theta(1 / y)(1-\delta) g_{0}\right],
\end{aligned}
$$

ce qui est équivalent à :

$$
\varphi_{0} \hat{r}_{t}^{L}=\varphi_{1} \hat{r}_{t}^{M}-\varphi_{2}^{\prime} \hat{y}_{t}-\varphi_{3}^{\prime} \sum_{n=0}^{+\infty} \beta^{n} \mathrm{E}_{t}\left\{\hat{y}_{t+n}\right\}
$$

avec :

$$
\begin{aligned}
\varphi_{2}^{\prime} & \equiv \omega r^{L} j_{0}+\varphi_{2}^{B}, \\
\varphi_{3}^{\prime} & \equiv \varphi_{3}^{B} .
\end{aligned}
$$

La transformation en quasi-différence $\left(\hat{r}_{t}^{L}-\beta \mathrm{E}_{t}\left\{\hat{r}_{t+1}^{L}\right\}\right)$ permet de simplifier la loglinéarisation et nous obtenons :

$$
\begin{aligned}
\varphi_{0} \hat{r}_{t}^{L}= & \varphi_{0} \beta \mathrm{E}_{t}\left\{\hat{r}_{t+1}^{L}\right\}+\varphi_{1}\left(\hat{r}_{t}^{M}-\beta \mathrm{E}_{t}\left\{\hat{r}_{t+1}^{M}\right\}\right) \\
& -\varphi_{2}^{\prime}\left(\hat{y}_{t}-\beta \mathrm{E}_{t}\left\{\hat{y}_{t+1}\right\}\right)-\varphi_{3}^{\prime} \hat{y}_{t} .
\end{aligned}
$$

Nous définissons $L \hat{L} R_{t}^{\prime} \equiv-\varphi_{3}^{\prime} \hat{y}_{t}$. Nous obtenons donc :

$$
\begin{aligned}
\varphi_{0} \hat{r}_{t}^{L}= & \varphi_{0} \beta \mathrm{E}_{t}\left\{\hat{r}_{t+1}^{L}\right\}+\varphi_{1}\left(\hat{r}_{t}^{M}-\beta \mathrm{E}_{t}\left\{\hat{r}_{t+1}^{M}\right\}\right) \\
& -\varphi_{2}^{\prime}\left(\hat{y}_{t}-\beta \mathrm{E}_{t}\left\{\hat{y}_{t+1}\right\}\right)+L \hat{L} R_{t}^{\prime} .
\end{aligned}
$$

\section{B.3 The capital buffer system}

La log-linéarisation de la CPO sur le taux des crédits dans le système de capital buffer est donnée par :

$$
\begin{aligned}
\hat{r}_{t}^{L}\left[r^{L}\right]\left[1-j_{0}-g_{0}\right]= & \hat{r}_{t}^{M}\left[r^{M}\right]\left[(\zeta /(\zeta-1))\left(1-k_{0}\right)\right]+\hat{y}_{t}[y]\left[-r^{L}(1 / y)\left(\omega j_{0}+\theta g_{0}\right)\right. \\
& \left.-(\zeta /(\zeta-1))\left(\omega(1 / y)(1-\eta) h_{0} j_{0}+\theta(1 / y) \delta g_{0}\right)\right] \\
& -\sum_{n=0}^{+\infty} \beta^{n+1} \mathrm{E}_{t}\left\{\hat{y}_{t+n}\right\}[y]\left[(\zeta /(\zeta-1)) r^{M}\left(\theta(1 / y)(1-\delta) g_{0}-\omega(1 / y) h_{0}(1-\eta) j_{0}\right)\right]
\end{aligned}
$$

ce qui est équivalent à :

$$
\varphi_{0} \hat{r}_{t}^{L}=\varphi_{1} \hat{r}_{t}^{M}-\varphi_{2}^{\prime \prime} \hat{y}_{t}-\varphi_{3}^{\prime \prime} \sum_{n=0}^{+\infty} \beta^{n} \mathrm{E}_{t}\left\{\hat{y}_{t+n}\right\}
$$

avec :

$$
\begin{aligned}
\varphi_{2}^{\prime \prime} & \equiv \omega\left(r^{L} j_{0}+Z(1-\eta) h_{0} j_{0}\right)+\varphi_{2}^{B}, \\
\varphi_{3}^{\prime \prime} & \equiv(1-\eta) \varphi_{3}^{A}+\varphi_{3}^{B} .
\end{aligned}
$$

La transformation en quasi-différence $\left(\hat{r}_{t}^{L}-\beta \mathrm{E}_{t}\left\{\hat{r}_{t+1}^{L}\right\}\right)$ permet de simplifier la log- 
linéarisation et nous obtenons :

$$
\begin{aligned}
\varphi_{0} \hat{r}_{t}^{L}= & \varphi_{0} \beta \mathrm{E}_{t}\left\{\hat{r}_{t+1}^{L}\right\}+\varphi_{1}\left(\hat{r}_{t}^{M}-\beta \mathrm{E}_{t}\left\{\hat{r}_{t+1}^{M}\right\}\right) \\
& -\varphi_{2}^{\prime \prime}\left(\hat{y}_{t}-\beta \mathrm{E}_{t}\left\{\hat{y}_{t+1}\right\}\right)-\varphi_{3}^{\prime \prime} \hat{y}_{t} .
\end{aligned}
$$

Nous définissons $L \hat{L} R_{t}^{\prime \prime} \equiv-\varphi_{3}^{\prime \prime} \hat{y}_{t}$. Nous obtenons donc :

$$
\begin{aligned}
\varphi_{0} \hat{r}_{t}^{L}= & \varphi_{0} \beta \mathrm{E}_{t}\left\{\hat{r}_{t+1}^{L}\right\}+\varphi_{1}\left(\hat{r}_{t}^{M}-\beta \mathrm{E}_{t}\left\{\hat{r}_{t+1}^{M}\right\}\right) \\
& -\varphi_{2}^{\prime \prime}\left(\hat{y}_{t}-\beta \mathrm{E}_{t}\left\{\hat{y}_{t+1}\right\}\right)+L \hat{L} R_{t}^{\prime \prime} .
\end{aligned}
$$

